\title{
A New Method of Area under the Absorbance-Wavelength Curve for Rats Total Metabolomic Pharmacokinetics from Yangxue Injection with Multicomponents
}

\author{
Lihong Zhang, Xiaojin Xiao, Zhenzhen Yang, Mengli Jiang, and Xiaodong Li \\ College of Pharmacy, Fujian University of Traditional Chinese Medicine, Fujian, Fuzhou 350122, China \\ Correspondence should be addressed to Xiaodong Li; lxdtcm@163.com
}

Received 13 July 2013; Accepted 2 August 2013

Academic Editor: Yunping Qiu

Copyright (C) 2013 Lihong Zhang et al. This is an open access article distributed under the Creative Commons Attribution License, which permits unrestricted use, distribution, and reproduction in any medium, provided the original work is properly cited.

To bridge the convergence between traditional Chinese medicine (TCM) and modern medicine originated from the West, a new method of area under the absorbance-wavelength curve (AUAWC) by spectrophotometer scanning was investigated and compared with HPLC method to explore metabolomic pharmacokinetics in rats. AUAWC and drug total concentration were obtained after Yangxue was injected to rats. Meanwhile, individual concentrations of sodium ferulate, tetramethylpyrazine hydrochloride, tanshinol sodium, and sodium tanshinone IIA sulfonate in plasma were determined by HPLC. Metabolomic profile of multicomponents plasma concentration time from AUAWC and that of individual components from HPLC were compared. The data from AUAWC had one-compartment model with mean area under concentration versus time (AUC) of $9370.58 \mathrm{~min} \cdot \mu \mathrm{g} / \mathrm{mL}$ and mean elimination half-life $\left(t_{1 / 2}\right)$ of $12.92 \mathrm{~min}$. The results by HPLC demonstrated that sodium ferulate and tetramethylpyrazine hydrochloride had one-compartment model with AUC of 6075.50 and $876.94 \mathrm{~min} \cdot \mu \mathrm{g} / \mathrm{mL}, t_{1 / 2}$ of 10.85 and $20.57 \mathrm{~min}$, respectively. Tanshinol sodium and sodium tanshinone IIA sulfonate showed two-compartment model, and AUC was 29.58 and 201.46 with $t_{1 / 2}$ of 1.76 and 16.90, respectively. The profiles indicated that method of AUAWC can be used to study pharmacokinetics of TCM with multicomponents and to improve its development of active theory and application in clinic combined with in vivo metabolomic profile of HPLC.

\section{Introduction}

For around 200 years, two very different systems of medicine have been used in Asia to cure diseases and keep people healthy. The local Asian one is based on traditional Chinese medicine (TCM) - herbal mixtures developed through observation and experience accumulated over thousands of years in China, but with unknown mechanisms of action [1]. On the other hand, modern medicine, which originated from the West, consists of chemically purified compounds that have been discovered through scientific investigation and tested in controlled clinical trials. These two phenomena confirmed the right saying in the career of traditional Chinese medicine that individual drug has its characteristic merits with good effectiveness, but compound formula has excellent clinical application with mixtures reasonable [2]. However, the most classical method, especially high performance liquid chromatography (HPLC), of metabolomic pharmacokinetics studies for western chemical drugs is effectively used to determine the drug metabolomic concentration in blood at different kinetic time [3-6]. It can explain the variation of the intensity of the pharmacological effects and explore many in vivo key issues during a pharmacokinetics study, such as absorption and distribution of chemical drugs, bioavailability, biotransformation and excretion pathway. As for TCM, only one or more effective components with known chemical structures in mixtures were selected as targets to investigate the pharmacokinetics by determining the individual metabolomic component concentration in the blood, which is the same as the method of western chemical 
drugs' pharmacokinetics studies [7-10]. Even if a single herb of TCM contains dozens or hundreds of kinds of chemical components [11, 12], not to mention the Chinese herbal formula $[13,14]$, some individual metabolomic components' in vivo tests cannot represent pharmacokinetics process of TCM with complicated multicomponents and cannot clarify the relationship between a variety of components and integral efficacy [15]. Therefore, the pharmacokinetics study of various individual metabolomic components is difficult to reasonably clarify the pharmacokinetics characteristics of TCM owing to its complexity. Up to now, many related theories and hypotheses about pharmacokinetics of TCM had been studied to explore its metabolomic behavior, but the substantial progresses have not been improved yet [16-21]. It is very important and urgent to establish reasonable analytical methods for metabolomic pharmacokinetics of TCM with multicomponents in order to improve its development of active theory and scientific application in clinic.

In this study, the method of area under the absorbancewavelength curve (AUAWC) was used to determine the total metabolomic contents of Yangxue injection in rats, which is composed of sodium ferulate, tetramethylpyrazine hydrochloride, tanshinol sodium, and sodium tanshinone IIA sulfonate and has effects on reducing blood viscosity, eliminating blood fat, and improving blood circulation. The previous results verified that the linear relationship between AUAWC and drug total concentration was good by the scanning of ultraviolet-visible spectrophotometer [22]. Therefore, the total drug contents of Yangxue injection could be determined through AUAWC of blood samples in rats, and the metabolomic profile could be obtained when the data in vivo were analyzed. Moreover, compared with the metabolomic profile from the HPLC method, the feasibility, rationality, and accuracy of the method of AUAWC used for TCM with multicomponents would be verified according to pharmacokinetics data from Yangxue injection. It will play an important role in the integration of TCM and western medicine in future pharmacokinetics research and clinical application.

\section{Materials and Methods}

2.1. Apparatus. Ultraviolet-visible spectrum scan was measured on a TU-1901 spectrophotometer (Beijing Purkinje General Instrument Co., Ltd.) with the use of a $1.0 \mathrm{~cm}$ quartz cell. Drug concentrations in plasma were measured on a LC20AT high performance liquid chromatograph (Shimadzu, Japan).

2.2. Reagents. Ferulic acid, tetramethylpyrazine hydrochloride, and tanshinol sodium reference substances were obtained from the National Institute for the Control of Pharmaceutical and Biological Products with batch numbers 110773-201012, 110855-200809, and 110817-200305, respectively. Sodium tanshinone IIA sulfonate reference substance was obtained from the National Institutes For Food and Drug Control with batch number 111605-201202. Yangxue injection was obtained from the Department of Pharmaceutics of
Fujian University of TCM. All other reagents were of HPLC grade, and the water used in the experiments was double distilled.

2.3. Animals. Healthy Sprague-Dawley rats (male), weighing 180 200 g, were obtained from SLAC Laboratory Animal (Shanghai, China, SCXK (HU) 2007-0005). All the rats were allowed to acclimate one week before treatment. All studies were in compliance with the Guidelines for the Care and Use of Laboratory Animals and approved by Institutional Animal Care and Use Committee in XBL-China.

2.4. Animal Administration and Sampling. 60 rats were randomly divided into ten groups based on different interval times. One group was intravenously injected $0.9 \%$ sodium chloride injection, and the others were injected Yangxue injection through the vein of rats tail. The rats were fasted overnight and injected a dose of $132.8 \mathrm{mg} / \mathrm{kg}$. Blood samples (about $2.0 \mathrm{~mL}$ ) from intraocular angular vein of rats were collected and placed into EDTA-2Na anticoagulant tubes at predetermined interval times $(5,10,20,30,60,90,120$, 180 , and $240 \mathrm{~min}$ ). Then, blood samples were centrifuged at $3800 \mathrm{rpm}$ for $10 \mathrm{~min}$, and the separated plasma samples were stored at $-20^{\circ} \mathrm{C}$ for next analysis.

\subsection{Method of AUAWC by Scanning of Ultraviolet-Visible Spectrophotometer}

2.5.1. Preparation of Calibration Standards. The stock solutions of mixture of ferulic acid $(1.0 \mathrm{mg} / \mathrm{mL})$, tetramethylpyrazine hydrochloride $(0.3 \mathrm{mg} / \mathrm{mL})$, tanshinol sodium $(0.25 \mathrm{mg} / \mathrm{mL})$, and sodium tanshinone IIA sulfonate $(0.11 \mathrm{mg} / \mathrm{mL})$ reference substances were freshly prepared in methanol and kept at $4^{\circ} \mathrm{C}$ before use, with a total concentration of $1.66 \mathrm{mg} / \mathrm{mL}$. Working solutions were prepared by diluting with methanol for further concentration series of $0.26,0.52,1.04,2.07,4.15,8.30,16.59$, and $33.18 \mu \mathrm{g} / \mathrm{mL}$.

2.5.2. Linearity. In accordance with the UV-visible spectrophotometry, methanol was used as a blank control. Full ultraviolet-visible spectrum scan to the above series of mixture standard solutions from $200 \mathrm{~nm}$ to $600 \mathrm{~nm}$ wavelength was carried out. The AUAWC of the total drugs was calculated by Origin software. An eight-point calibration curve was constructed by plotting the AUAWC against the total concentration. The linearity of the calibration curve was evaluated by linear regression analysis.

2.5.3. Sample Preparation and Test. $400 \mu \mathrm{L}$ plasma sample was precisely diluted with methanol to $4 \mathrm{~mL}$, vortexed for 2 minutes, and centrifuged at $3800 \mathrm{r} / \mathrm{min}$ for 10 minutes. Then, supernatant was used for a full ultraviolet-visible spectrum scanning from $200 \mathrm{~nm}$ to $600 \mathrm{~nm}$ wavelength with methanol as a blank control.

2.5.4. Pharmacokinetics Study of Yangxue Injection. Absorbance values of full wavelength scan were put into Origin software, and the AUAWC of the plasma samples at each interval 
time was calculated. When the average values of AUAWC of drug group subtract those of the control group, respectively, the increased AUAWC caused by total metabolomic drugs was obtained correspondingly and then substituted into the linear equation to get the total metabolomic drug concentration in vivo. The pharmacokinetics models (oneversus two-compartment) were compared according to the AIC rule, with minimum AIC values being regarded as the best representation of the blood concentration-time dynamic data. A compartmental model of Yangxue injection in rats was proposed by the computer program $3 \mathrm{p} 97$.

\subsection{Method of HPLC}

2.6.1. Chromatographic Conditions. The HPLC system used was a Shimadzu HPLC, consisting of two LC-10A pumps, a SIL-10AF autoinjector, a SPD-M10A photodiode array detector, and a SCL-10A system controller. A Vision HT C $\mathrm{H}_{18}$ $(4.6 \mathrm{~mm} \times 250 \mathrm{~mm}, 5 \mu \mathrm{m})$ column was used. The mobile phase consisted of methanol and $0.5 \%$ acetic acid $(28: 72 \mathrm{v} / \mathrm{v})$ that was filtered and degassed prior to use, while sodium ferulate and tetramethylpyrazine hydrochloride were tested. The mobile phase of tanshinol sodium was consisted of methanol and $0.2 \%$ phosphoric acid $(15: 85 \mathrm{v} / \mathrm{v})$, and the methanol and phosphate buffer $(60: 40 \mathrm{v} / \mathrm{v})$ was used to separate sodium tanshinone IIA sulfonate. Isocratic elution at $30^{\circ} \mathrm{C}$ at a flow rate of $1.0 \mathrm{~mL} / \mathrm{min}$. Ultraviolet detection of sodium ferulate, tetramethylpyrazine hydrochloride, tanshinol sodium, and sodium tanshinone IIA sulfonate was carried out at 322, 296, 280 , and $271 \mathrm{~nm}$, respectively.

2.6.2. Preparation of Calibration Standards and Quality Control Samples. The standard stock solutions of ferulic acid/tetramethylpyrazine hydrochloride $(340 / 237 \mu \mathrm{g} / \mathrm{mL})$, tanshinol sodium $(207 \mu \mathrm{g} / \mathrm{mL})$, and sodium tanshinone IIA sulfonate $(108 \mu \mathrm{g} / \mathrm{mL})$ were freshly prepared in methanol and kept at $4^{\circ} \mathrm{C}$ before use, respectively. Working solutions of ferulic acid/tetramethylpyrazine hydrochloride were prepared by diluting with methanol for further concentration series of $136 / 94.8,54.4 / 37.9,21.8 / 15.2,8.70 / 6.07,3.48 / 2.43$, $1.39 / 0.971,0.557 / 0.388,0.223 / 0.155$, and $0.0891 / 0.0621 \mu \mathrm{g} / \mathrm{mL}$. Similarly, working solutions of tanshinol sodium were prepared at concentration series of $82.8,33.1,13.2,5.30,2.12$, $0.848,0.339$, and $0.136 \mu \mathrm{g} / \mathrm{mL}$ and solutions of sodium tanshinone IIA sulfonate at $6.91,2.76,1.11,0.442,0.177$, $0.0708,0.0283$, and $0.0113 \mu \mathrm{g} / \mathrm{mL}$. Calibration work solutions were prepared by spiking $200 \mu \mathrm{L}$ of the appropriate working solutions to $100 \mu \mathrm{L}$ of blank plasma. The quality control (QC) samples including low, middle, and high concentrations of standards were prepared in the same way as the calibration standards. All calibration work solutions and QC samples were stored at $-20^{\circ} \mathrm{C}$ until analysis.

2.6.3. Sample Preparation. $200 \mu \mathrm{L}$ of methanol was precisely added into a $100 \mu \mathrm{L}$ portion of plasma sample. Then, the mixtures were vortexed for 2 minutes and centrifuged at

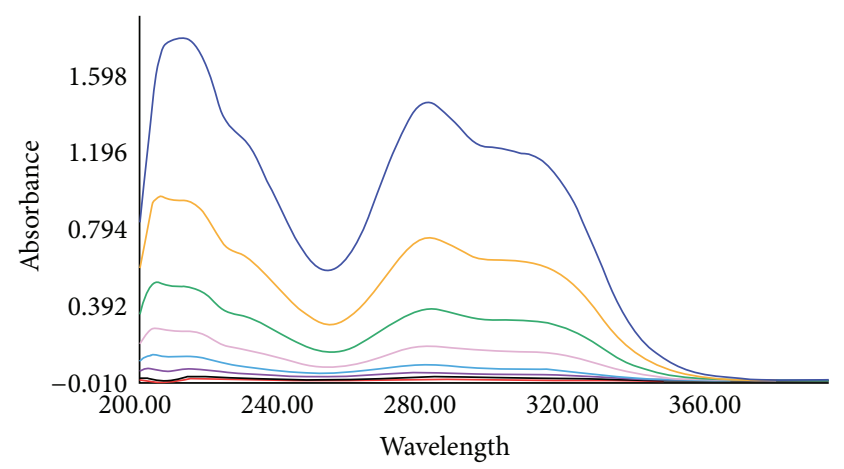

FIGURE 1: Full spectrum scan of mixed standard substance. (Concentration increased successively from bottom to top).

TABLE 1: The average values of AUAWC $(n=6)$.

\begin{tabular}{lcc}
\hline Time $(\mathrm{min})$ & AUAWC of plasma & AUAWC of drugs \\
\hline 0 & 91.21 & \\
5 & 247.23 & 156.02 \\
10 & 223.65 & 132.44 \\
20 & 204.83 & 113.62 \\
30 & 178.01 & 86.80 \\
60 & 121.02 & 29.81 \\
90 & 94.03 & 2.82 \\
120 & 92.99 & 1.78 \\
180 & 81.09 & -10.12 \\
240 & 78.95 & -12.26 \\
\hline
\end{tabular}

TABLE 2: Pharmacokinetics mean parameters of 4 components and potential metabolites in rat after Yangxue injection administration.

\begin{tabular}{lc}
\hline Parameters & Mean value \\
\hline$C_{0} / \mu \mathrm{g} \cdot \mathrm{mL}^{-1}$ & 502.75 \\
$t_{1 / 2}(\mathrm{Ke}) / \mathrm{min}$ & 12.92 \\
$\mathrm{Ke} / \mathrm{min}^{-1}$ & 0.05 \\
$\mathrm{AUC} / \mu \mathrm{g} \cdot \mathrm{mL}^{-1} \cdot \mathrm{min}$ & 9370.58 \\
$\mathrm{CL}(\mathrm{s}) / \mathrm{L} \cdot \mathrm{min}^{-1}$ & 0.014 \\
$V(\mathrm{c}) / \mathrm{L}$ & 0.26 \\
\hline
\end{tabular}

$15,000 \mathrm{rpm}$ for 10 minutes, and the aliquot $(30 \mu \mathrm{L})$ of the supernatant was submitted to the HPLC system.

\subsubsection{Method Validation}

Specificity. The specificity of the method was investigated by comparing chromatograms of blank rat plasma, each standard of ferulic acid, tetramethylpyrazine hydrochloride, tanshinol sodium, and sodium tanshinone IIA sulfonate, the blank plasma spiked with each standard, and plasma samples obtained from rats after administration. There should be no interference from endogenous or exogenous materials observed at the retention time of the four components.

Linearity. A nine-point calibration curve with final concentrations of $90.7 / 63.2,36.3 / 25.3,14.5 / 10.1,5.80 / 4.04,2.32 / 1.62$, 
TABLE 3: The correlation coefficients and linear range of linear regression equations.

\begin{tabular}{lccc}
\hline Compound & Equation & Correlation coefficients & Linear range $(\mu \mathrm{g} / \mathrm{mL})$ \\
\hline Ferulic acid & $Y=147126 X-41291$ & 0.9999 & $0.0594 \sim 90.7$ \\
Tetramethylpyrazine hydrochloride & $Y=73946 X+8923.8$ & 0.9999 & $0.104 \sim 63.2$ \\
Tanshinol sodium & $Y=26194 X-1882.6$ & 0.9999 & $0.565 \sim 138$ \\
Sodium tanshinone IIA sulfonate & $Y=110717 X+3682.3$ & 0.9999 & $7.55 \times 10^{-3} \sim 4.61$ \\
\hline
\end{tabular}

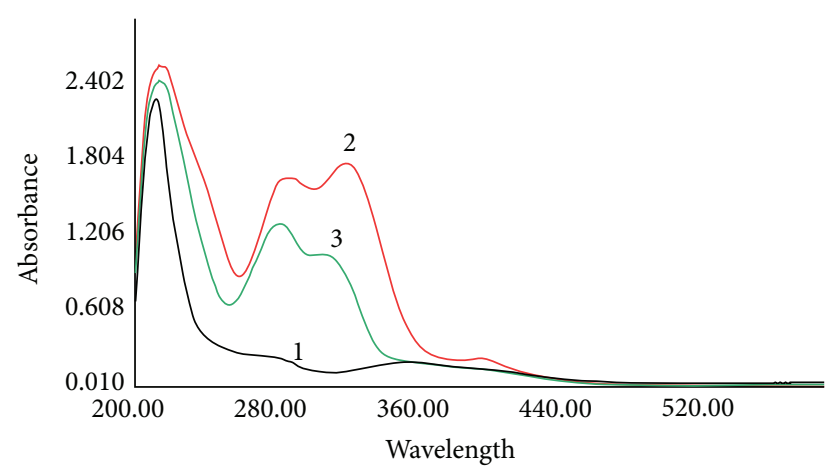

Figure 2: Scanning spectrum of blank plasma (1) and plasma sample obtained at 5 and 30 min with 4 components and potential metabolites after i.v. administration ( 2 and 3 ).

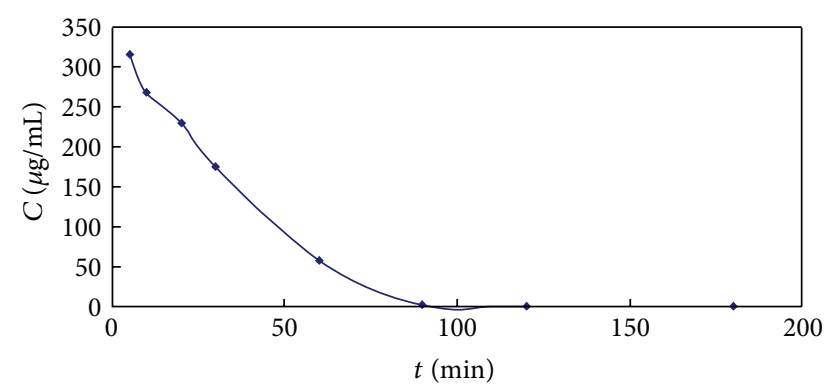

Figure 3: Profile of total drugs concentration versus time curve in rats plasma with 4 components and potential metabolites after Yangxue injection administration.

$0.928 / 0.647,0.371 / 0.259,0.149 / 0.104$, and $0.0594 / 0.0414 \mu \mathrm{g} /$ $\mathrm{mL}$ was constructed by plotting the peak area against the concentrations of ferulic acid/ tetramethylpyrazine hydrochloride, respectively. Similarly, the final concentrations of tanshinol sodium were 55.2, 22.1, 8.83, 3.53, 1.41, 0.565, 0.226, and $0.0904 \mu \mathrm{g} / \mathrm{mL}$, and those of sodium tanshinone IIA sulfonate were $4.61,1.84,0.737,0.295,0.118,0.0472,0.0189$, and $0.00755 \mu \mathrm{g} / \mathrm{mL}$. The linearity of the calibration curve was evaluated by linear regression analysis.

Recovery. The absolute recovery of ferulic acid/tetramethylpyrazine hydrochloride from rats plasma was calculated at concentrations of $0.371 / 0.259,2.32 / 1.62$, and $14.5 / 10.1 \mu \mathrm{g} / \mathrm{mL}$ by comparing the peak area of extracted samples with ones in which the compounds were spiked directly to methanol. The low, middle, and high concentrations of tanshinol sodium were $0.226,1.41$, and $8.83 \mu \mathrm{g} / \mathrm{mL}$ and sodium tanshinone IIA sulfonate with $0.118,0.737$, and $4.61 \mu \mathrm{g} / \mathrm{mL}$. Recoveries at
TABLE 4: The extraction recovery of 4 compounds in rat plasma $(n=$ $5)$.

\begin{tabular}{lccc}
\hline Compound & $\begin{array}{c}\text { Concentration } \\
(\mu \mathrm{g} / \mathrm{mL})\end{array}$ & $\begin{array}{c}\text { Recovery } \\
(\%)\end{array}$ & $\begin{array}{c}\text { RSD } \\
(\%)\end{array}$ \\
\hline \multirow{3}{*}{ Ferulic acid } & 14.5 & 109.95 & 8.57 \\
& 2.32 & 98.42 & 1.88 \\
\hline Tetramethylpyrazine & 0.371 & 113.24 & 3.65 \\
hydrochloride & 10.1 & 123.01 & 15.34 \\
& 1.62 & 106.22 & 3.79 \\
Tanshinol sodium & 0.259 & 104.83 & 1.68 \\
\hline \multirow{2}{*}{ Sodium tanshinone IIA } & 8.83 & 90.28 & 1.04 \\
sulfonate & 1.41 & 85.22 & 0.53 \\
& 0.226 & 86.53 & 0.55 \\
\hline
\end{tabular}

three QC concentration levels for plasma were examined at least five times.

Precision and Accuracy. The precision and accuracy of the method were determined by analyzing QC samples at three different concentrations the same as recovery experiment. Intraday precision and accuracy were assessed from replicate analyses $(n=5)$ of QC samples at each concentration level on the same day. Precision was determined by calculating the RSD for each replicate, and accuracy was assessed by calculating the percentage bias from the nominal concentration. The precisions were required to be within $\pm 15 \%$ relative standard deviation and the accuracy to be within $\pm 15 \%$ bias from the nominal values.

2.6.5. Calculations and Statistics. 3p97 software was employed to analyze the plasma concentration-time kinetic data. The area under the concentration-time curve (AUC) from zero to the last time point, elimination half-life time $\left(t_{1 / 2}\right)$, volume of distribution $(\mathrm{Vd})$, and plasma clearance $(\mathrm{Cl})$ were all obtained.

\section{Results and Discussion}

\subsection{The Results of AUAWC}

3.1.1. Linearity. From Figure 1, there existed a good linear relationship between AUAWC and total drug concentrations with the same composition and proportion 
TABLE 5: The precision and accuracy of 4 compounds in rat plasma $(n=5)$.

\begin{tabular}{|c|c|c|c|c|}
\hline \multirow{2}{*}{ Compound } & \multirow{2}{*}{ Concentration $(\mu \mathrm{g} / \mathrm{mL})$} & \multirow{2}{*}{ Precision (RSD, \%) } & \multicolumn{2}{|c|}{ Accuracy } \\
\hline & & & Accuracy (\%) & RSD (\%) \\
\hline \multirow{3}{*}{ Ferulic acid } & 14.5 & 1.13 & 95.05 & 4.39 \\
\hline & 2.32 & 0.53 & 109.59 & 1.67 \\
\hline & 0.371 & 0.09 & 105.36 & 3.58 \\
\hline \multirow{3}{*}{ Tetramethylpyrazine hydrochloride } & 10.1 & 4.95 & 110.00 & 4.95 \\
\hline & 1.62 & 0.48 & 100.05 & 0.48 \\
\hline & 0.259 & 0.80 & 99.60 & 0.80 \\
\hline \multirow{3}{*}{ Tanshinol sodium } & 8.83 & 1.01 & 95.59 & 1.01 \\
\hline & 1.41 & 0.53 & 100.12 & 0.53 \\
\hline & 0.226 & 0.55 & 99.97 & 0.55 \\
\hline \multirow{3}{*}{ Sodium tanshinone IIA sulfonate } & 4.61 & 1.85 & 105.51 & 2.34 \\
\hline & 0.737 & 4.62 & 109.95 & 4.81 \\
\hline & 0.118 & 3.23 & 108.62 & 3.25 \\
\hline
\end{tabular}

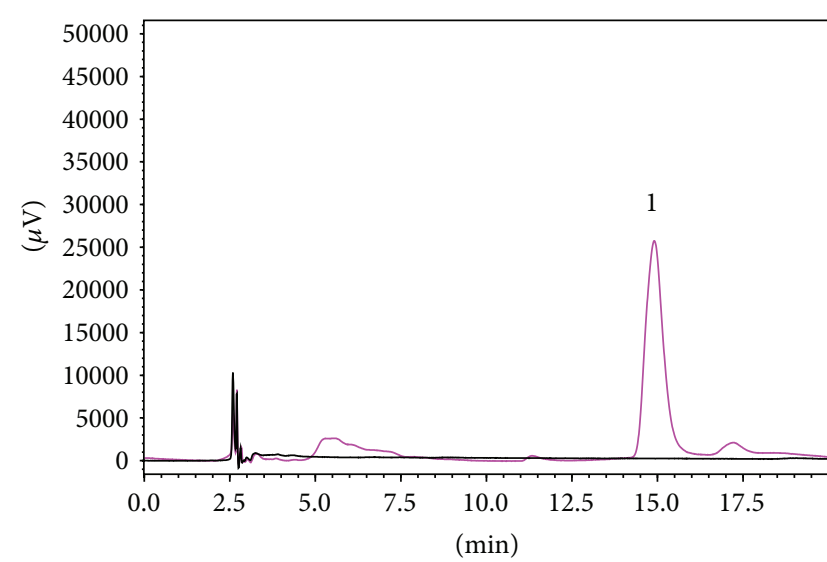

(a)

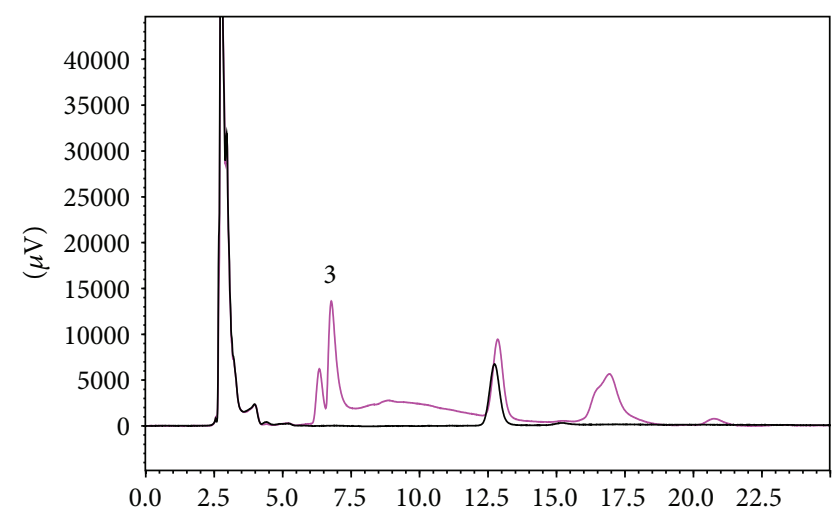

(c)

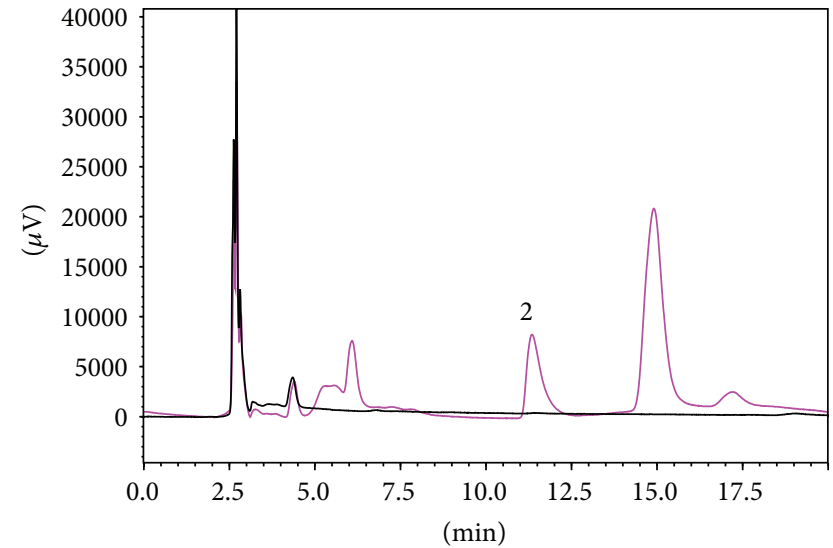

(b)

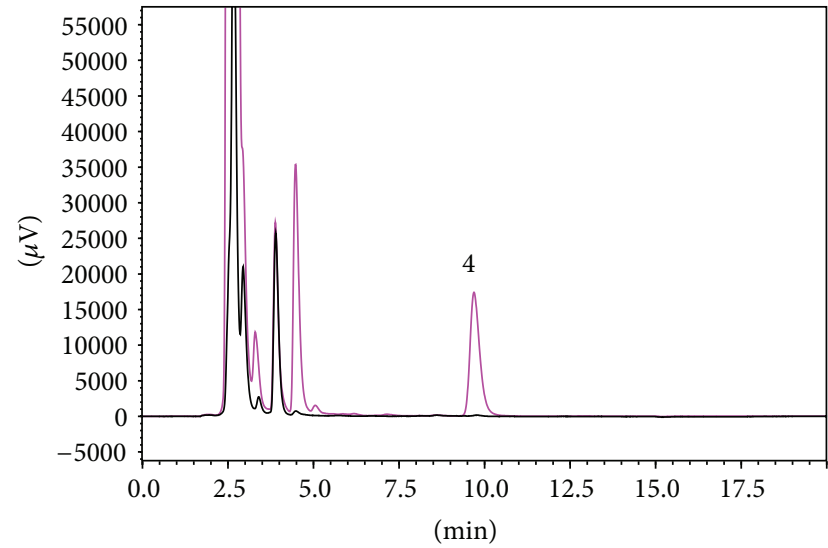

(d)

FIGURE 4: Chromatograms of blank plasma (black) and plasma sample at 30 minute (pink). Peaks: 1, sodium ferulate; 2 , tetramethylpyrazine hydrochloride; 3 , tanshinol sodium; 4, sodium tanshinone IIA sulfonate.

as Yangxue injection. The result showed a good correlation coefficient $(r=0.9994)$ for mixed drugs over the total concentration range from 0.26 to $33.18 \mu \mathrm{g} / \mathrm{mL}$. The regression equation is AUAWC $=4.8936 \mathrm{C}+1.3210$ $(r=0.9994)$.
3.1.2. Pharmacokinetics Study of Yangxue Injection. Full wavelength scan of different rat plasmas presented in Figure 2. Absorbance values of full wavelength scan were put into Origin software, and the AUAWC of the control group and drug groups at different interval times was calculated. 
The mean AUAWC values of plasma samples and the increased AUAWC caused by drugs and potential metabolites were provided in Table 1 . When the latter was substituted into the linear equation, the total metabolomic drugs concentrations in vivo were obtained. The profile of concentration versus time kinetic curve in rats plasma after Yangxue injection $(132.8 \mathrm{mg} / \mathrm{kg}$, i.v.) administration was shown in Figure 3. Main pharmacokinetics parameters of Yangxue injection in rats were presented in Table 2.

\subsection{The Results of HPLC}

\subsubsection{Method Validation}

Specificity. Black curves in Figure 4 showed chromatograms of blank plasma after protein precipitation, illustrating the baseline without any disturbing peaks in the chromatograms of plasma samples. No interference or other metabolites were coeluted with target components. Pink curves in Figure 4 showed chromatograms of plasma samples after Yangxue injection administration at 30 minute, which were marked and had the similar places with those of standards' chromatograms of ferulic acid, tetramethylpyrazine hydrochloride, tanshinol sodium, and sodium tanshinone IIA sulfonate, respectively.

Linearity. As shown in Table 3, the results demonstrated good correlation coefficients $(r \geq 0.999)$ for ferulic acid, tetramethylpyrazine hydrochloride, tanshinol sodium, and sodium tanshinone IIA sulfonate over the concentration range from separately prepared analytical compounds.

Recovery. The extraction recovery of ferulic acid, tetramethylpyrazine hydrochloride, tanshinol sodium, and sodium tanshinone IIA sulfonate in rats plasma was presented in Table 4.

Precision and Accuracy. The precision and accuracy of ferulic acid, tetramethylpyrazine hydrochloride, tanshinol sodium, and sodium tanshinone IIA sulfonate in rats plasma were presented in Table 5.

3.2.2. Pharmacokinetics Study of Sodium Ferulate, Tetramethylpyrazine Hydrochloride, Tanshinol Sodium, and Sodium Tanshinone IIA Sulfonate. The results demonstrated that both sodium ferulate and tetramethylpyrazine hydrochloride appeared to have one-compartment model in rats, but tanshinol sodium and sodium tanshinone IIA sulfonate appeared to have two-compartment model. The profile of concentration versus time kinetic curves and pharmacokinetics parameters of the above four components in rat plasma after administration were showed in Figure 5 and Tables 6 and 7. The pharmacokinetics data showed that the individual mean plasma concentration of all four components was rapidly distributed and eliminated in $120 \mathrm{~min}$ after intravenous administration to rats.
TABLE 6: Pharmacokinetics parameters of sodium ferulate and tetramethylpyrazine hydrochloride.

\begin{tabular}{lcc}
\hline Parameters & Sodium ferulate & $\begin{array}{c}\text { Tetramethylpyrazine } \\
\text { hydrochloride }\end{array}$ \\
\hline$C_{0} / \mu \mathrm{g} \cdot \mathrm{mL}^{-1}$ & 388.25 & 29.55 \\
$t_{1 / 2}(\mathrm{Ke}) / \mathrm{min}$ & 10.85 & 20.57 \\
$\mathrm{Ke} / \mathrm{min}^{-1}$ & 0.064 & 0.034 \\
$\mathrm{AUC} / \mu \mathrm{g} \cdot \mathrm{mL}^{-1} \cdot \mathrm{min}$ & 6075.50 & 876.94 \\
$\mathrm{CL}(\mathrm{s}) / \mathrm{L} \cdot \mathrm{min}^{-1}$ & 0.013 & 0.027 \\
$V(\mathrm{c}) / \mathrm{L}$ & 0.206 & 0.812 \\
\hline
\end{tabular}

TABle 7: Pharmacokinetics parameters of tanshinol sodium and sodium tanshinone IIA sulfonate.

\begin{tabular}{lcc}
\hline Parameters & Tanshinol sodium & $\begin{array}{c}\text { Sodium tanshinone } \\
\text { IIA sulfonate }\end{array}$ \\
\hline$A(\mu \mathrm{g} / \mathrm{mL})$ & 88.29 & 25.17 \\
$B(\mu \mathrm{g} / \mathrm{mL})$ & 6.19 & 1.49 \\
$C_{0}(\mu \mathrm{g} / \mathrm{mL})$ & 94.48 & 26.66 \\
$\alpha(1 / \mathrm{min})$ & 6.35 & 0.152 \\
$\beta(1 / \mathrm{min})$ & 0.395 & 0.041 \\
$\mathrm{AUC} / \mu \mathrm{g} \cdot \mathrm{mL}^{-1} \cdot \mathrm{min}$ & 29.58 & 201.46 \\
$\mathrm{CL}(\mathrm{s}) / \mathrm{L} \cdot \mathrm{min}^{-1}$ & 0.676 & 0.044 \\
$t_{1 / 2, \alpha}(\mathrm{min})$ & 0.109 & 4.55 \\
$t_{1 / 2, \beta}(\mathrm{min})$ & 1.76 & 16.90 \\
\hline
\end{tabular}

\section{Conclusions}

There existed a good linear relationship between AUAWC and total concentration of four components composed with the same proportion as Yangxue injection. Both methods of AUAWC and HPLC were feasible and accurate, which will bring a feasible idea and pathway to pharmacokinetics' studies of TCM with multicomponents. From Tables 2, 6, and 7, the value of $C_{0}$ from AUAWC, 502.75, was similar to the $C_{0}$ sum of individual ferulic acid, tetramethylpyrazine hydrochloride, tanshinol sodium, and sodium tanshinone IIA sulfonate, 538.94. AUC in AUAWC was 9370.58, more about 2187 than that obtained from HPLC, 7183.48, with the sum of individual four components, which was mainly related to new metabolites in rats when compared with the metabolomic profiles from AUAWC and HPLC methods. Also, the value of $t_{1 / 2}$ including $t_{1 / 2}(\mathrm{Ke})$ in one-compartment model or $t_{1 / 2, \alpha}$ and $t_{1 / 2, \beta}$ in two-compartment model was all not more than 21 minutes, which indicated a quick distribution and fast excretion in rats that were injected Yangxue injection and had a good accordance with [23-26]. It can be concluded that the method of AUAWC can be conveniently used for the in vivo pharmacokinetics study on TCM with multicomponents. If combined with the method of conventional western medicine pharmacokinetics study, such as HPLC or HPLC-MS, to analyze and determine the new certain metabolites, the active theory will be elucidated easily and the clinical application will be performed reasonably for TCM compound with multicomponents. Similarly, the 


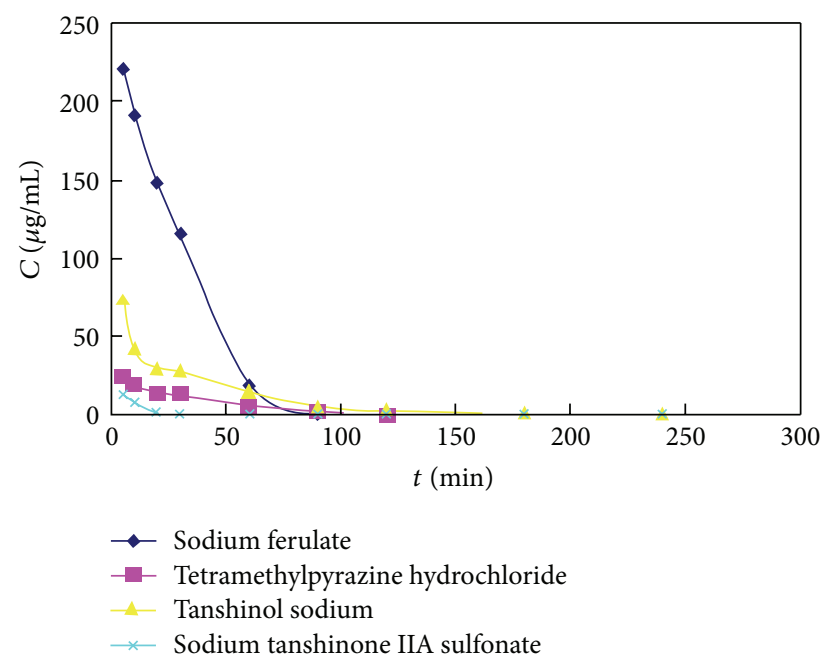

FIGURE 5: The profile of concentration versus time kinetic curves after administration.

method of AUAWC is useful for the pharmacokinetics study of individual drug or multi-component chemical drugs. It will play an important role in the integration and convergence of TCM and Western medicine.

\section{Acknowledgment}

This study was supported by technology platform construction fund from Fujian Provincial Department of Science and Technology (2010Y2004) and fund of Keji Chen (CKJ2012022).

\section{References}

[1] Q. L. Liang, G. A. Luo, J. Q. Zou et al., "Strategy and technical support system for developing new TCM drugs," World Science and Technology, vol. 10, no. 3, pp. 1-7, 2008.

[2] S. P. Wang, Y. Y. Hu, W. Tan et al., "Compatibility art of traditional Chinese medicine: from the perspective of herb pairs," Journal of Ethnopharmacology, vol. 143, no. 2, pp. 412423, 2012

[3] C. M. Wang, G. T. Lin, Z. Wang et al., "Determination of imatinib in rat plasma by HPLC and study of its pharmacokinetics," Chinese Pharmaceutical Journal, vol. 48, no. 4, pp. 293-296, 2013.

[4] H. L. Chen, W. P. Zhang, F. Y. Yang, X. Y. Wang, W. C. Yang, and H. W. Dang, "Pharmacokinetic interaction of pioglitazone hydrochloride and atorvastatin calcium in Beagle dogs," Acta Pharmaceutica Sinica, vol. 48, no. 5, pp. 741-745, 2013.

[5] J. J. Qi, W. Li, L. S. Zhao et al., "Pharmacokinetic interaction between aminophylline and metronidazole in rats," Chinese Pharmaceutical Journal, vol. 48, no. 1, pp. 63-67, 2013.

[6] J. J. Qi, X. H. Chen, K. S. Bi, W. Li, and X. Chen, "A study of pharmacokinetic interaction between isosorbide mononitrate injection and sodium ferulate for injection in rats," Chinese Journal of Hospital Pharmacy, vol. 33, no. 3, pp. 377-381, 2013.
[7] G. Chen, F. Lu, L. J. Xu et al., "The anti-diabetic effects and pharmacokinetic profiles of berberine in mice treated with JiaoTai-Wan and its compatibility," Phytomedicine, vol. 20, no. 10, pp. 780-786, 2013

[8] Y. G. Sun, Y. F. Du, K. Yang et al., "A comparative study on the pharmacokinetics of a traditional Chinese herbal preparation with the single herb extracts in rats by LC-MS/MS method," Journal of Pharmaceutical and Biomedical Analysis, vol. 81-82, pp. 34-43, 2013.

[9] H. X. Zhu, Z. L. Qian, F. He et al., "Novel pharmacokinetic studies of the Chinese formula Huang-Lian-Jie-Du-Tang in MCAO rats," Phytomedicine, vol. 20, no. 10, pp. 767-774, 2013.

[10] T. Li, Y. W. Wang, Y. L. Wang et al., "Development of an SPE-HPLC-MS method for simultaneous determination and pharmacokinetic study of bioactive constituents of Yu Ping Feng San in rat plasma after oral administration," Journal of Ethnopharmacology, vol. 145, pp. 784-792, 2013.

[11] C. Xu and Z.-T. Wang, "Chemical constituents from roots of Andrographis paniculata," Acta Pharmaceutica Sinica, vol. 46, no. 3, pp. 317-321, 2011.

[12] Y. P. Shen, X. X. Zhong, Z. J. Yu, C. Zhou, H. Yang, and X. Jia, "Chemical constituents of Toona Sinensis leaves," Chinese Pharmaceutical Journal, vol. 48, no. 1, pp. 22-24, 2013.

[13] L. Chen, D. Wang, J. Wu, B. Yu, and D. Zhu, "Identification of multiple constituents in the traditional Chinese medicine formula GuiZhiFuLing-Wan by HPLC-DAD-MS/MS," Journal of Pharmaceutical and Biomedical Analysis, vol. 49, no. 2, pp. 267-275, 2009.

[14] J. C. Zhang, G. Y. Chen, C. R. Han et al., "Chemical constituents in active fraction of Gankang Formula," Chinese Traditional Patent Medicine, vol. 35, no. 2, pp. 316-320, 2013.

[15] Q. Zheng, P.-F. Yue, B. Wu, P.-Y. Hu, Z.-F. Wu, and M. Yang, "Pharmacokinetics comparative study of a novel Chinese traditional herbal formula and its compatibility," Journal of Ethnopharmacology, vol. 137, no. 1, pp. 221-225, 2011.

[16] W. Y. Tao, X. Xu, X. Wang et al., "Network pharmacologybased prediction of the active components and potential targets of Chinese herbalRadix Curcumaeformula for application to cardiovascular disease," Journal of Ethnopharmacology, vol. 145, pp. 1-10, 2013.

[17] S. Li and B. Zhang, "Traditional Chinese medicine network pharmacology: theory, methodology and application," Chinese Journal of Natural Medicines, vol. 11, no. 2, pp. 110-120, 2013.

[18] S. S. Dou, R. H. Liu, P. Jiang et al., "System biology and its application in compound recipe of traditional Chinese medicine study," World Science and Technology, vol. 10, no. 2, pp. 116-121, 2008.

[19] H.-P. Hao, C.-N. Zheng, and G.-J. Wang, "Thoughts and experimental exploration on pharmacokinetic study of herbal medicines with multiple-components and targets," Acta Pharmaceutica Sinica, vol. 44, no. 3, pp. 270-275, 2009.

[20] S. X. You, C. J. Mao, Y. Cao et al., "Serum pharmacology of Chinese compound recipe huanglian injection," Lishizhen Medicine and Materia Medica Research, vol. 23, no. 8, pp. 1873$1875,2012$.

[21] X. Huang and P. Ren, "Medical innovation of post syndrom and treatment pharmacokinetics: a study on holism-guided reductionism," Journal of Traditional Chinese Medicine, vol. 54, no. 5, pp. 365-368, 2013. 
[22] X. D. Li, S. Y. Li, L. H. Zhang, X. Xiao, C. Hou, and Z. Yang, "Linear relationship between area under absorbancewavelength curve and drug concentration and its application in TCM pharmacokinetics," Journal of Fujian University of TCM, vol. 22, no. 6, pp. 26-31, 2012.

[23] Y. Zou, J.-C. Song, and H. Liu, "Pharmacokinetic study of ferulic acid in compound angelica powder for injection," Chinese Pharmaceutical Journal, vol. 41, no. 24, pp. 1885-1887, 2006.

[24] X. M. Chen, X. W. Wang, J. Luo et al., "Pharmacokinetic studies of Xuebijing injection in rats," Chinese Journal of Pharmaceutical Analysis, vol. 32, no. 5, pp. 744-748, 2012.

[25] Z. M. Xia, L. S. Wang, B. L. Lai et al., "Pharmacokinetics investigation of Xiongbing microemulsion in rabbit," Chinese Journal of Experimental Traditional Medical Formulae, vol. 17, no. 11, pp. 119-121, 2011.

[26] Y. Shi, X. Y. Li, T. Y. Wang et al., "Pharmacokinetics and pharmacodynamics studies of Tanshinone IIA and tanshinone IIA sulfonate," Chinese Journal of Pharmaceutics, vol. 7, no. 3, pp. 143-153, 2009. 

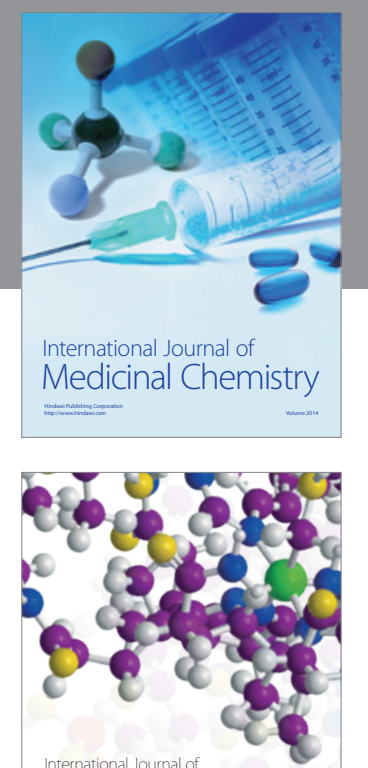

\section{Carbohydrate} Chemistry

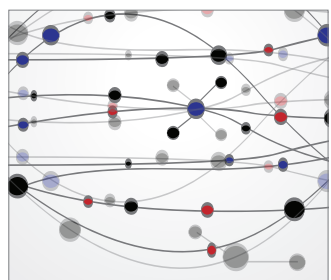

The Scientific World Journal
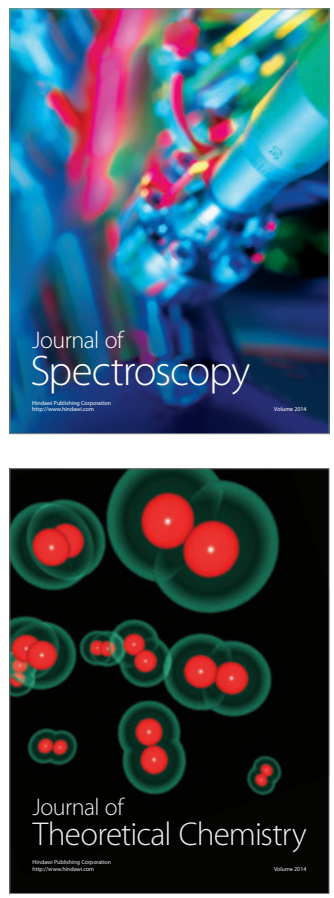
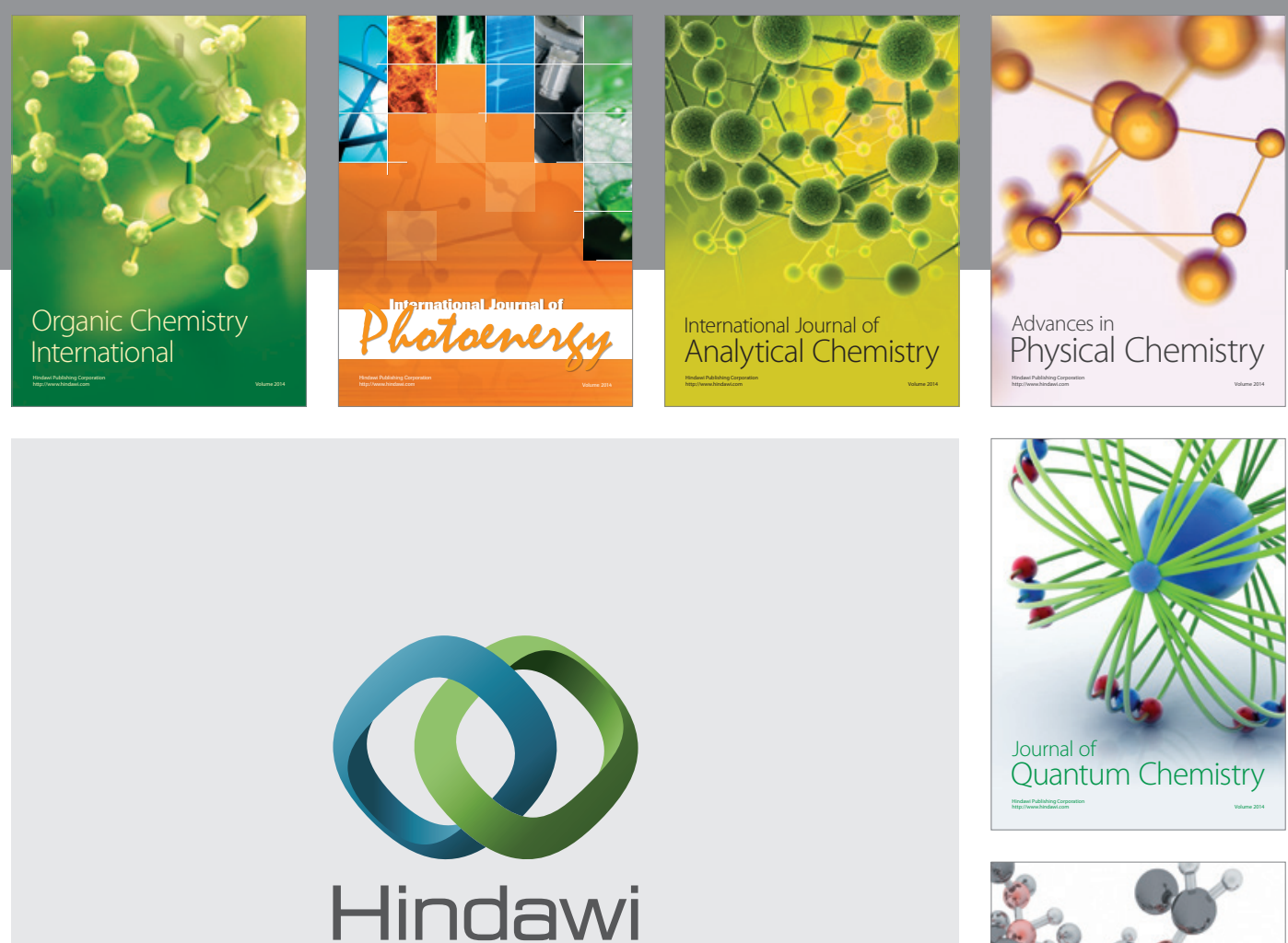

Submit your manuscripts at

http://www.hindawi.com

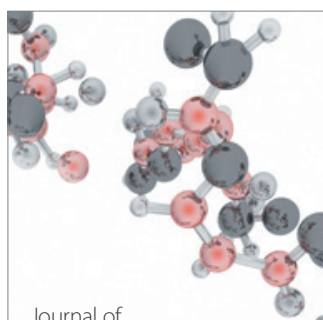

Analytical Methods

in Chemistry

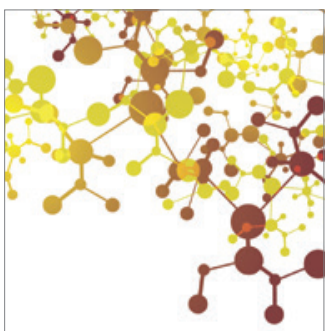

Journal of

Applied Chemistry

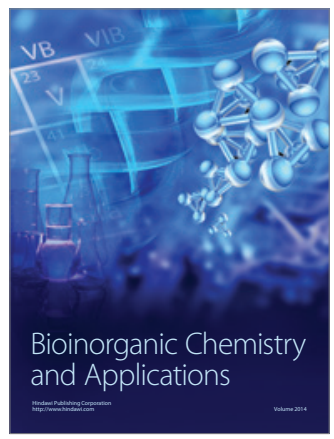

Inorganic Chemistry
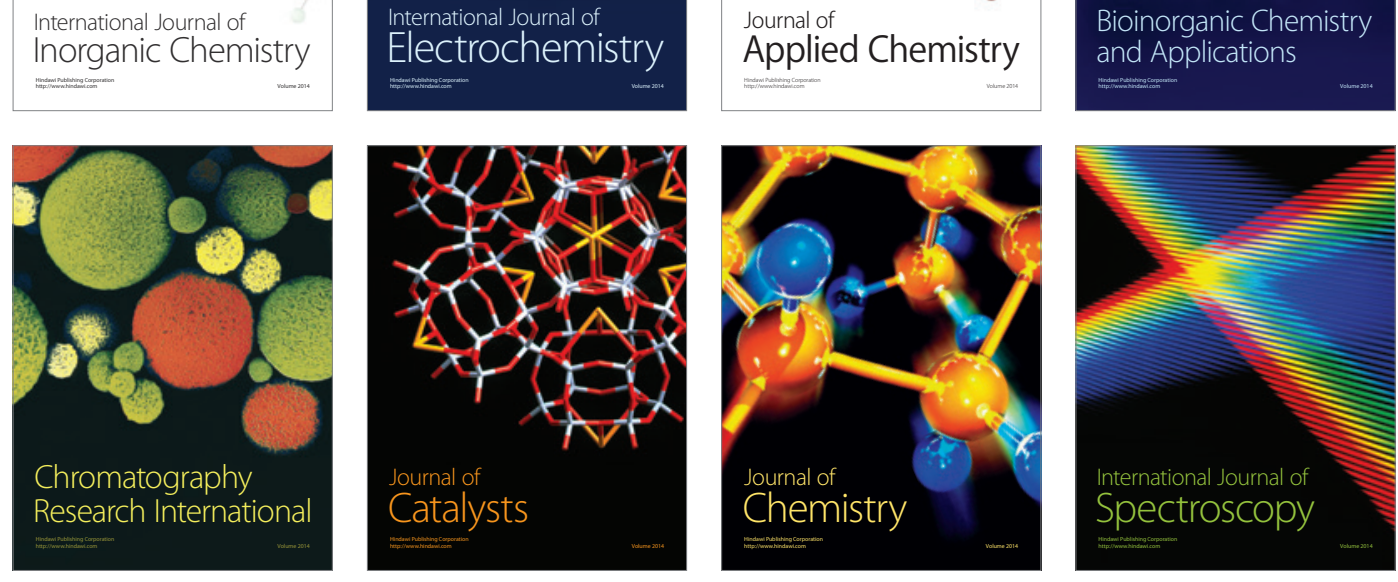